\title{
AUTOMATIC COASTLINE DETECTION IN NON-LOCALLY FILTERED TANDEM-X DATA
}

\author{
Michael Schmitt ${ }^{(1)}$, Lingyun Wei ${ }^{(1)}$, Xiao Xiang Zhu ${ }^{(1,2)}$ \\ (1) Helmholtz Young Investigators Group SiPEO, Technische Universitaet Muenchen (TUM), \\ Arcisstr. 21, 80333 Munich, Germany \\ (2) Remote Sensing Technology Institute (IMF), German Aerospace Center (DLR), \\ Oberpfaffenhofen, 82234 Wessling, Germany
}

\section{INTRODUCTION}

The detection of coastlines in SAR imagery has been studied for more than two decades now. Whereas the first works were based on the exploitation of amplitude imagery and the corresponding need to deal with speckle noise [1, 2, 3], with the ERS-1/2 tandem configuration also coherence maps started to be used as input $[4,5]$. Based on the insights gained on these experiments, later the authors began to exploit both amplitude and coherence imagery simultaneously [6, 7], finally giving way to the first approach using the original complex SAR data for statistically motivated coastline extraction [8].

In any case, one of the core problems for coastline detection from SAR datasets is the comparably high level of noise, which degrades the grayscale input imagery, be it amplitude or coherence. In order to solve this deficiency, an abundance of filtering techniques have been proposed over the years, among which the most sophisticated are based on the recently introduced non-local paradigm. Since Deledalle et al. proposed the NL-InSAR algorithm designed for interferometric multilooking in 2011 [9], novel denoising qualities can be achieved. In [10] and [11], it was already shown that the final DEM to be created during the German TanDEM-X mission [12] can be improved significantly by a specifically optimized implementation of NL-InSAR.

This paper proposes a fully automatic framework for coastline extraction from non-locally filtered TanDEM-X data based on unsupervised active contours. It is therefore structured into two main parts: First, the non-local filtering framework will be described. Second, the active contours algorithm for coastline detection will be described. Finally, some preliminary experimental results processed from an interferometric TerraSAR-X dataset showing the skerry coast in the area of Stockholm, Sweden, will be demonstrated.

\section{NON-LOCAL FILTERING OF TANDEM-X DATASETS}

Non-local filters have been shown to provide strong noise reduction while still well retaining fine structures such as linear features or point targets. The basic principle of non-local filters is to exploit the high degree of redundancy in any natural image: similar patches of every target patch usually can be found many times in the same image. Rather than averaging pixels in a pre-defined local neighborhood (e.g. rectangular box-car windows), or spatially connected adaptive regions, non-local filters consider pixels in a significantly larger search area and weight them according to a certain patch-based similarity measure. This way, the number of pixels to be averaged for noise reduction is much higher, while the similaritybased nonlocal averaging avoids the smoothing of edges or fine structures, and thus helps resolution preservation.

Adapted to the case of InSAR multilooking, the NLInSAR filter provides not only a non-locally filtered interferometric phase, but simultaneously also despeckled amplitude and less biased coherence estimation. These resulting nonlocal estimates can serve as highly accurate input data for coastline extraction.

In this work, the nonlocal processing of the TerraSAR-X stripmap data is carried out using the algorithm developed in $[10,11]$.

\section{UNSUPERVISED ACTIVE CONTOURS FOR COASTLINE DETECTION}

The first experiments of active contours for shoreline detection in very high resolution TerraSAR-X data were demonstrated in [13], with focus on the analysis of large lakes and manual initialization of the contour model.

In contrast, the method for coastline detection employed in this paper is based on the so-called Chan-Vese Active Contours model [14] with automatic initialization. This ChanVese segmentation algorithm, which - in contrast to many other segmentation methods - is not based on any kind of edge detection, was inspired by the Mumford-Shah model [15]. It basically consists of the minimization of the func- 
tional

$$
\begin{aligned}
& F\left(c_{1}, c_{2}, C\right)= \\
& \mu\|C\|+\int_{\Omega_{1}=\omega}\left(f(x, y)-c_{1}\right)^{2} d x d y \\
& +\int_{\Omega_{2}=\Omega-\omega}\left(f(x, y)-c_{2}\right)^{2} d x d y .
\end{aligned}
$$

In this equation, $f(x, y)$ denotes the input image, $C$ denotes the curve necessarily describing the boundary of a closed set, and $\Omega, \Omega_{1}$ and $\Omega_{2}$ denote the domain to be segmented, the part inside the curve $C$ and the part outside the curve $C$, respectively. $c_{1}$ and $c_{2}$ are two unknown constants.

In order to solve this optimization problem, the level set technique introduced by Osher and Sethian [16] is used. Instead of manipulating $C$ explicitly, it is replaced by the relationship

$$
C=\{(x, y) \in \Omega \mid \phi(x, y)=0\} .
$$

Thus, the functional of (1) becomes

$$
\begin{aligned}
& F\left(c_{1}, c_{2}, \phi\right)= \\
& \mu \int_{\Omega}\|\nabla H(\phi)\|+\int_{\Omega}\left(f(x, y)-c_{1}\right)^{2} H(\phi) d x d y \\
& +\int_{\Omega}\left(f(x, y)-c_{2}\right)^{2}(1-H(\phi)) d x d y,
\end{aligned}
$$

where $H(\cdot)$ is the Heaviside function. Now, in order to minimize $F$, its partial derivatives with respect to the unknowns have to be set to zero. Finally, the solution can be found by updating $c_{1}, c_{2}$ and $\phi$ recursively. A nice introduction including more details about Chan-Vese active contours and their implementation can be found in [17].

In combination with a checkerboard-like initialization (cf. Fig. 1), this technique allows a fully automatic segmentation even of heterogeneous land/water regions, not requiring any manual interaction.

\section{Postprocessing}

After the segmentation result is achieved from the active contours procedure, post-processing is carried out in order to get rid of small inland lakes and other outliers using an algorithm based on morphological reconstruction [18]. An example for this step is shown in Fig. 2. Afterwards, the segment borders are extracted using the Moore-Neighbor tracing algorithm modified by Jacob's stopping criterion. The resulting dataset is supposed to represent all coastlines present in the scene, defined as the line separating solid land mass and islands from the open sea. Shorelines of inland lakes or islands in inland lakes are not considered in the context of this work.

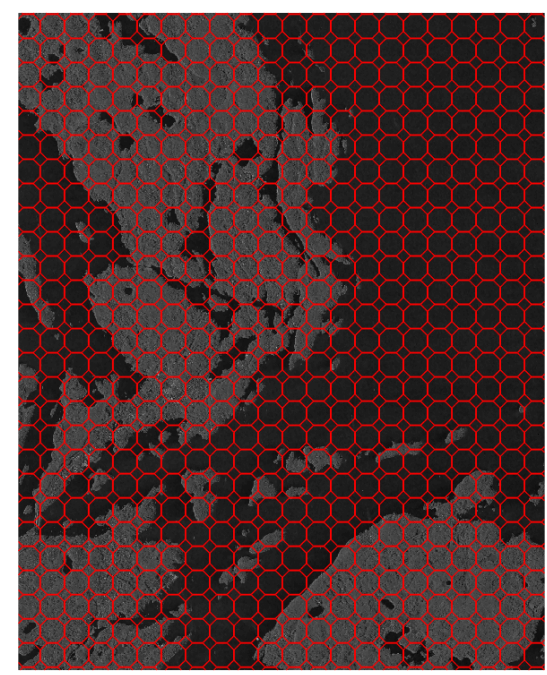

Fig. 1. Initial mask for the Chan-Vese active contours algorithm.

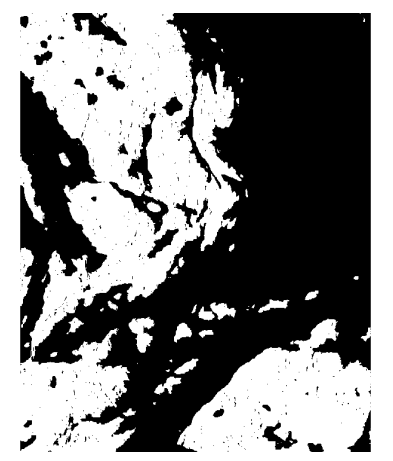

(a)

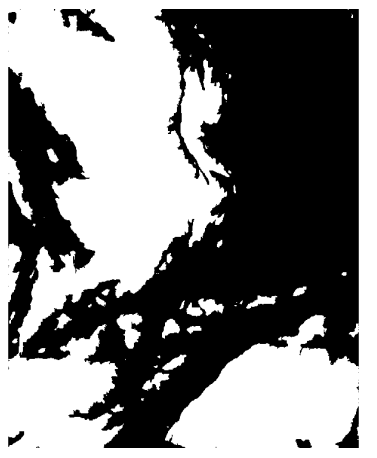

(b)
Fig. 2. (a) Result of active contours segmentation. (b) Result of morphological filling. All outliers and inland lakes are removed, whereas fine structures, fjords and islands are kept.

\section{TEST DATASET}

For the first experiments shown in this paper, an interferometric TerraSAR-X dataset acquired at August 31, 2014, and September 11, 2014, was used. The data were acquired in stripmap mode with a resolution of approximately $3.3 \mathrm{~m}$ in azimuth and $1.2 \mathrm{~m}$ in slant range. The interferometric image pair was processed using NL-InSAR as described in Section 2, and the resulting despeckled amplitude image was used for further processing. Afterwards, the image was resampled to a ground sampling distance of $2.0 \mathrm{~m}$ during SRTM-based geocoding.

\section{EXPERIMENTS AND RESULTS}

First results of the coastline extraction based on the nonlocally filtered TerraSAR-X amplitude image and Chan-Vese 
Active Contours can be seen in Fig. 3. It can be seen that all obvious coastlines - even of small islands - were detected, while the inland lakes were ignored. On the other hand, the fjord touching the upper border of the scene was detected as well. For evaluation purposes, a reference dataset was manually extracted from high-resolution optical imagery. Figure 4 shows the detected coastlines in comparison to these reference coastlines by displaying displacement vectors. It can be noted that sometimes the detected coastline over-, sometimes under-estimates the land extent. In addition, it can be seen that a fjord in the center of the scene was missed since obviously the canal between inner fjord and open sea is too small to be detected in the available resolution. The median of the displacement vectors is 7.5 meters (i.e. 3-4 pixels).

\section{DISCUSSION}

Although the active contours-based coastline detection procedure works well on non-locally filtered TerraSAR-X amplitude imagery, the quantitative results still show room for significant further improvement. The main error sources can be identified as follows:

- The geocoding of the TerraSAR-X data was carried out making use of the SRTM digital terrain model, which leads to certain remaining geocoding errors.

- Manual extraction of reference coastlines from optical imagery is also not free from potential errors.

- Effects caused by high and low tide can lead to errors, if SAR dataset and reference dataset are not acquired simultaneously.

In addition to these potential error causes referring to the comparability of SAR-derived coastlines and reference data, it has to be mentioned that the parameter $\mu$ as well as the radii of the circles in the initial contours influence the active contours segmentation to a certain extent. For the experiments in this paper, both have been chosen empirically, but adaptive selection would be advisable and therefore is part of future work.

\section{ACKNOWLEDGMENT}

The authors would like to thank Gerald Baier for nonlocal processing of the TanDEM-X stripmap data. This work is supported by the Helmholtz Association under the framework of the Young Investigators Group "SiPEO” (VH-NG-1018, www.sipeo.bgu.tum.de).

\section{REFERENCES}

[1] Jong-Sen Lee and Igor Jurkevich, "Coastline detection and tracing in SAR images," IEEE Transactions on Geoscience and Remote Sensing, vol. 28 , no. 4 , pp. 662-668, 1990.
[2] D. Zhang, L. Van Gool, and A. Osterlinck, "Coastline detection from SAR images," in Proceedings of IEEE International Geoscience and Remote Sensing Symposium, 1994, pp. 2134-2136.

[3] Xavier Descombes, Miguel Moctezuma, Henri Maitre, and Jean-Paul Rudant, "Coastline detection by a Markovian segmentation on SAR images," Signal Processing, vol. 55, no. 1, pp. 123-132, 1996.

[4] Marcus Schwäbisch, Susanne Lehner, and Norbert Winkel, "Coastline extraction using ERS SAR interferometry," in Proceedings of ERS Symposium, 1997, pp. 1049-1053.

[5] S. Dellepiane, R. De Laurentiis, and F. Giordano, "Coastline extraction from SAR images and a method for the evaluation of the coastline precision," Pattern Recognition Letters, vol. 25, no. 13, pp. 1461-1470, 2004.

[6] Andreas Niedermeier, Danielle Hoja, and Susanne Lehner, "Topography and morphodynamics in the German Bight using SAR and optical remote sensing data," Ocean Dynamics, vol. 55, no. 2, pp. 100-109, 2005.

[7] Anna Wendleder, Birgit Wessel, Achim Roth, Markus Breunig, Klaus Martin, and Susanne Wagenbrenner, "TanDEM-X water indication mask: generation and first evaluation results," IEEE Journal of Selected Topics in Applied Earth Observations and Remote Sensing, vol. 6, no. 1, pp. 171-179, 2013.

[8] Fabio Baselice and Giampaolo Ferraioli, "Unsupervised coastal line extraction from SAR images," IEEE Geoscience and Remote Sensing Letters, vol. 10, no. 6, pp. 1350-1354, 2013.

[9] Charles-Alban Deledalle, L. Denis, and Florence Tupin, "NL-InSAR: Nonlocal interferogram estimation," IEEE Transactions on Geoscience and Remote Sensing, vol. 49, no. 4, pp. 1441-1452, 2011.

[10] Xiaoxiang Zhu, Richard Bamler, Marie Lachaise, Fathalrahman Adam, Yilei Shi, and Michael Eineder, "Improving TanDEM-X DEMs by non-local InSAR filtering," in Proceedings of European Conference on Synthetic Aperture Radar, 2014, pp. 1125-1128.

[11] Xiaoxiang Zhu, Marie Lachaise, Fathalrahman Adam, Yilei Shi, Gerald Baier, Michael Eineder, and Richard Bamler, "Beyond the $12 \mathrm{~m}$ TanDEM-X DEM - quality improvement by non-local InSAR filtering," IEEE Journal of Selected Topics in Applied Earth Observations and Remote Sensing, Submitted.

[12] G. Krieger, A. Moreira, H. Fiedler, I. Hajnsek, M. Werner, M. Younis, and M. Zink, "TanDEM-X: a satellite formation for high-resolution SAR interferometry," IEEE Transactions on Geoscience and Remote Sensing, vol. 45, no. 11, pp. 3317-3341, 2007.

[13] Thomas Hahmann and Birgit Wessel, "Surface water body detection in high-resolution TerraSAR-X data using active contour models," in Proceedings of 8th European Conference on Synthetic Aperture Radar, 2010, pp. 897-900.

[14] T.F. Chan and L.A. Vese, "Active contours without edges," IEEE Transactions on Image Processing, vol. 10, no. 2, pp. 266-277, 2001.

[15] David Mumford and Jayant Shah, "Optimal approximations by piecewise smooth functions and associated variational problems," Communications on Pure and Applied Mathematics, vol. 42, no. 5, pp. 577-685, 1989.

[16] Stanley Osher and James A. Sethian, "Fronts propagating with curvature-dependent speed: algorithms based on Hamilton-Jacobi formulations," Journal of Computational Physics, vol. 79, no. 1, pp. 1249, 1988.

[17] Pascal Getreuer, "Chan-Vese segmentation," Image Processing On Line, vol. 2, pp. 214224, 2012.

[18] P. Soille, Morphological Image Analysis: Principles and Applications, Springer-Verlag, 1999. 


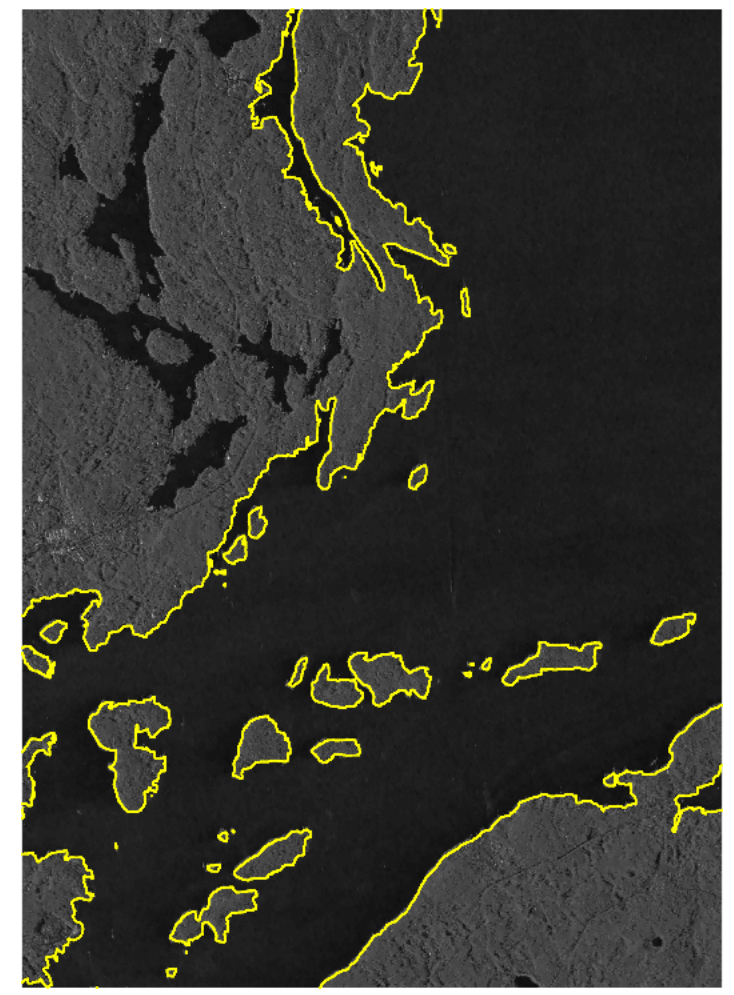

(a)

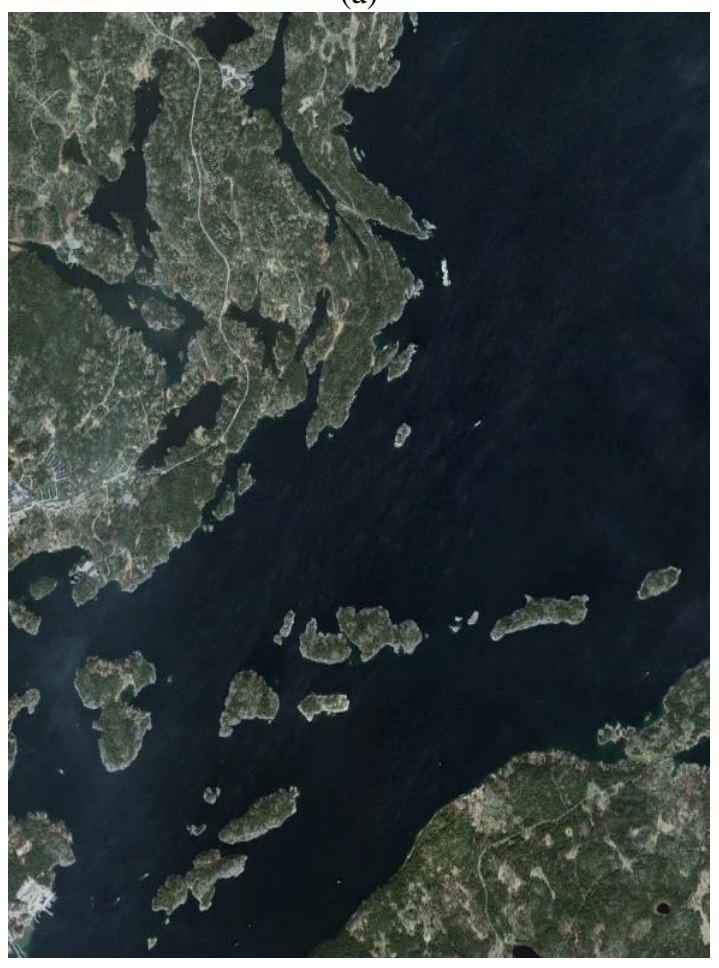

(b)

Fig. 3. (a) First result of coastline detection on the skerry coast near Stockholm, Sweden, based on non-local filtering and Chan-Vese Active Contours. (b) Corresponding optical image (C)2015 Google Earth)

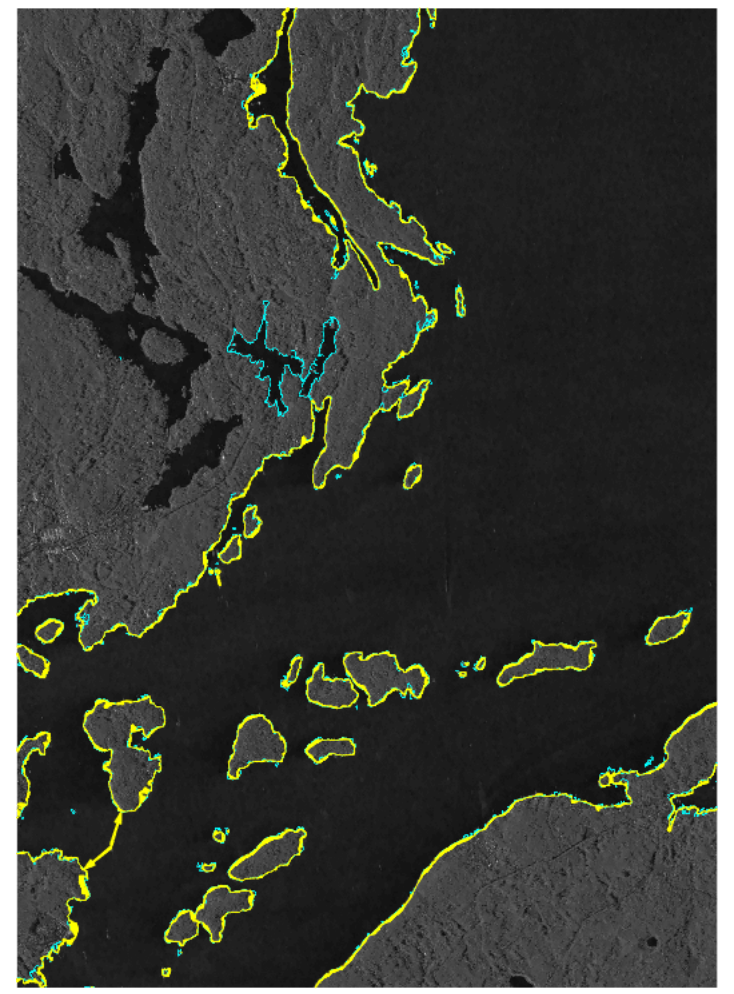

(a)

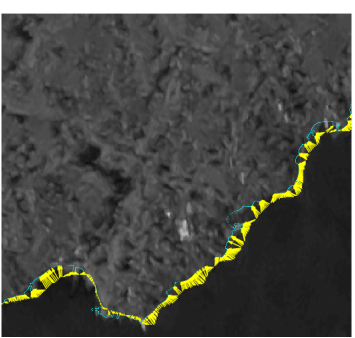

(b)

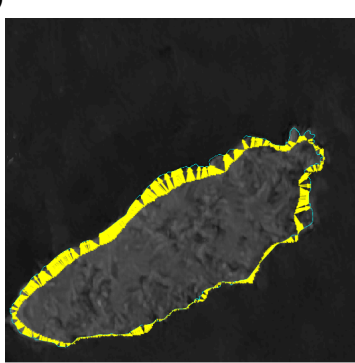

(c)
Fig. 4. (a) Displacement vectors between detected coastline (yellow) and reference coastline (cyan). (b) Detail view for exemplary subset of land shore, (c) detail view for exemplary island shore. 